\title{
Implementación de estrategias de Aprendizaje Centrado en el Estudiante en un curso de Ingeniería
}

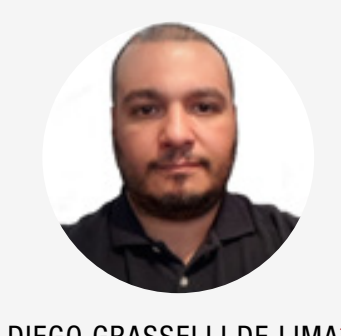

DIEGO GRASSELLI DE LIMA*

\section{Implementation of Student-Centered Learning in an Engineering Course}

Recibido: 8 de abril de 2019 | Aprobado: 18 de junio de 2019

\section{Resumen}

Históricamente la educación se ha caracterizado por desarrollarse en función del docente siendo este el centro de atención en el proceso de aprendizaje. El campo de la Ingeniería no escapa a esta situación. De acuerdo a las características de nuestra sociedad actual y el perfil de los estudiantes, esta práctica no es la más adecuada. El objetivo que nos proponemos es mostrar un relato de experiencia a partir de la implementación de estrategias del Aprendizaje Centrado en el Estudiante que mejoran la forma de aprender de nuestros alumnos y, a la vez, la forma en que el docente enseña, ya no como el actor principal en el proceso de enseñanza/aprendizaje, sino como el facilitador y promotor de aprendizajes auténticos y competenciales que transformen dicho proceso en una actividad más placentera para ambos.

Palabras clave: aprendizaje centrado en el estudiante; aprendizaje activo; metodología; estilos de aprendizaje.

\begin{abstract}
Education has historically been characterized by its dependence on the teaching role, who is the center of the learning process. This is true also in the engineering field. Due to the characteristics of the current society and the students' profile, today that kind of approach is not considered as the most suitable. We aim to demonstrate that Learning Processes focused on students should improve the way our students learn and should impact on the way our teachers work. These ones are not considered as the main actors of the teaching/learning process, on the contrary, they are seen as facilitators and promoters of true learning, as the responsible that this process would turn into a more enjoyable one for both parties.
\end{abstract}

Keywords: Student-Centered Learning; active learning; methodology; learning styles

\section{Introducción}

Históricamente hemos caracterizado a la educación en función del docente, siendo este el personaje central del proceso de aprendizaje. Si hiciéramos una encuesta probablemente las personas describirían un aula como un lugar con un profesor asumiendo el rol de disertante, de pie frente a los alumnos. En esta típica escena hay dos roles bien definidos: alguien que tiene el conocimiento y alguien que lo recibe, y la comunicación no es bidireccional, sino solamente unidireccional (desde el docente hacia los alumnos). Al tratar esta relación entre profesor y alumno como sujeto y objeto de la educación, López (2007) resalta que debemos dejar de lado la idea de una educación bancaria, término acuñado por Freire, ya que

\footnotetext{
* Licenciado en Administración y Análisis de Sistemas; Maestría en Docencia Universitaria; Certificación de Educador Internacional de Ingeniería (IGIP). Auxiliar Docente de la Universidad Tecnológica Nacional (UTN) - Facultad Regional de Buenos Aires. Para contactar al autor: diego.grasselli@gmail.com
}

ISSN (en línea): 1814-4152 / Sitio web: http://cuaderno.pucmm.edu.do CÓMO CITAR: Grasselli, D. (2019). Implementación de estrategias de Aprendizaje Centrado en el Estudiante en un curso de Ingeniería. Cuaderno de Pedagogía Universitaria, Vol. 16, n. ${ }^{\circ} 32$, julio-diciembre, pp. 70-81 
esta metodología no reconoce la figura del alumno como centro del proceso. A pesar de los cambios que la educación ha sufrido en las últimas décadas, sigue siendo el modelo que encontramos con más frecuencia en el ámbito universitario.

En la educación de la ingeniería este modelo de enseñanza tradicional se hace evidente muy frecuentemente en nuestras universidades aun hoy en día. Ante este panorama, Cukierman (2018) hace una pregunta relevante y que los docentes deberíamos responder: ¿qué es más importante, lo que enseñamos nosotros o lo que aprenden nuestros alumnos? De seguro, surgirían distintas respuestas, casi todas resaltando la importancia de lo que enseñamos, pero, y si los alumnos no aprenden, ¿de qué sirve todo lo que enseñamos? A partir de esta inquietud, Cukierman (2018) ofrece una guía para los docentes de ingeniería que desean mejorar sus prácticas, proponiendo algunas ventajas de la utilización del aprendizaje centrado en el estudiante. El autor resalta que esta metodología despierta gran interés y los docentes manifiestan genuinos deseos de implementarla, aunque en la mayoría de los casos no logran cambiar su modelo tradicional ya que necesitarían más herramientas y orientación para hacerlo.

Considerar al estudiante como foco del proceso de aprendizaje no es algo nuevo, es el mismo aprendizaje activo concebido por teóricos de gran impacto como Dewey, Carl Rogers, Jean Piaget, Lev Vygotsky y el tan conocido Brunner con el constructivismo de los años 80 y 90 del pasado siglo XX. Todos ellos indagaron y ofrecieron grandes aportes sobre cómo aprende el alumno. Los mismos principios impulsados por estos autores, pero ahora con la etiqueta de Aprendizaje Centrado en el Estudiante es una tendencia relativamente nueva que está ganando importancia en los últimos años a través de distintas publicaciones. Una de ellas es el estudio de Hernández, Salgado, y Sandoval (2007), quienes fomentan la importancia de impulsar el aprendizaje centrado en el estudiante para facilitar a los alumnos la posibilidad de construcción del conocimiento basado en sus intereses. Otro estudio es el de Bergmann (2018), quien propone un equilibrio entre la enseñanza tradicional y el aprendizaje centrado en el estudiante como la mejor estrategia a seguir.

Cambiar de paradigma no es tan fácil porque se requiere que los docentes dejen la posición de ser el centro del proceso de enseñanza y pasen a ser facilitadores del mismo. Esta resistencia es, de cierta forma, natural y se debe a la dificultad lógica de salir de la zona de confort representada por nuestro vasto conocimiento y la experticia que nos marca. Corroborando esta dificultad que soporta la innovación, López (2007, p. 31) enfatiza que este cambio es "un camino muy largo, difícil y tortuoso", por lo cual conlleva "formarse en actitudes, destrezas y hábitos, crear climas constructivos, afrontar conflictos, conocer procesos, prever y superar resistencias, manejar estrategias" (De la Torre, citado por López, 2007, p. 31).

Dada esta necesidad de cambio, de renovación de una enseñanza tradicional con la lección magistral como método privilegiado, nuestro objetivo con este artículo es presentar la implementación de estrategias y recursos del modelo de aprendizaje centrado en el estudiante y con ello motivar al docente a dejar el rol protagónico y tornarse en una especie de mentor de sus alumnos, convertir el aula en un espacio donde los estudiantes sean el centro de las actividades y dueños de su proceso de aprendizaje. Por lo cual presentaremos algunas actividades y ejemplos prácticos del Aprendizaje Centrado en el Estudiante, metodología activa en la que ellos construyen su propio aprendizaje y alcanzan un dominio más profundo de su disciplina.

En la primera parte de este documento describiremos el modelo de aprendizaje centrado en el estudiante como metodología privilegiada en la educación superior; los diferentes roles que intervienen en el proceso y la importancia del cambio de concepción del docente frente a esta metodología. También, presentaremos la importancia de identificar los estilos de aprendizaje del alumno y la necesidad de adaptar el contenido a ser enseñado de acuerdo a las características de los estudiantes con la finalidad de optimizar dicho aprendizaje. En la segunda parte describiremos algunos recursos y estrategias utilizados en el aprendizaje centrado en el estudiante. La tercera parte está dedicada a presentar ejemplos concretos de aplicación de actividades y recursos que bien pueden utilizarse en otros contextos. Por último, concluimos con una motivación a la innovación.

\section{El Aprendizaje Centrado en el Estudiante}

En el sistema actual de educación, a pesar de todas las innovaciones que se han propuesto a 
través de la tecnología, los rediseños curriculares y los enfoques didácticos, el docente sigue ocupando un rol protagónico por considerar que posee el conocimiento y, por tanto, se lo debe trasmitir al alumno. Según Castillo y Cabrerizo (2005), es muy común todavía observar en las aulas que la explicación expositiva es el método más utilizado por los docentes, sea por desconocimiento o por la falta de interés en aplicar una metodología apropiada a su materia. Esta práctica termina generando indiferencia y apatía por parte de los alumnos y, como consecuencia, los lleva a que su atención no esté en lo que se está explicando, produciendo muchas veces un bajo rendimiento o, incluso, la deserción académica. Una adecuación metodológica por parte del docente, que incluya estrategias y actividades acordes a su materia, puede llegar a crear en el alumno una motivación por un aprendizaje enriquecido con recursos y medios que lograrán llevarlo a la madurez y autonomía de su proceso de aprendizaje, y más que todo, a adquirir las competencias necesarias para su eficacia laboral.

Escribano y del Valle (2008) comentan que, a lo largo de la historia, facilitar el aprendizaje del alumno es una preocupación que siempre ha estado presente. Sin embargo, dado el desarrollo tecnológico y las características de los aprendices de este siglo, el rol del profesor se ha ampliado, surgiendo nuevas funciones, como, por ejemplo, la del tutor. Hoy al docente se le pide que, más allá de dominar el contenido, también tenga el conocimiento de nuevos métodos y estrategias didácticas acordes a la asignatura, a las competencias a desarrollar y a las características del grupo implicado en el aprendizaje. A razón de eso, el docente se ve compelido a implementar nuevas metodologías, teniendo que repensar la relación docente-estudiante-contenido. Acerca de eso, Riverón Portela, citado por Manzanares y Palomares (2008), comenta que el profesor debe ser un tutor que pueda guiar sus alumnos a aprender, convirtiéndose en motivador de situaciones de aprendizaje más que en facilitador de charlas magistrales.

En la actualidad, en las instituciones superiores hay una tendencia a cuestionar cómo se enseña y cómo se aprende, o sea, el método está ganando importancia, todo con el fin de mejorar la calidad del aprendizaje universitario. De ahí que este nuevo rol de tutor que debe asumir el docente logra cada vez más relevancia, ya que los estudiantes tendrán mayor motivación para aprender en la medida en que el docente mantenga una relación cercana y más horizontal con ellos y sea innovador en las estrategias que implementa. (Escribano y del Valle, 2008).

Desde la perspectiva de los estudiantes, Weimer (2002) comenta que el aprendizaje centrado en el estudiante (ACE) está ganando gran relevancia, y es que en este modelo se pueden aplicar distintas metodologías. El autor considera que en la enseñanza tradicional los alumnos son aprendices pasivos y con una relación vertical con respecto al docente, lo que provoca que no asuman la total responsabilidad sobre su aprendizaje, por lo cual, este autor resalta la importancia de implementar metodologías que den a los alumnos la "responsabilidad" por su aprendizaje. Sin embargo, es necesario que el docente estimule la motivación del alumno con relación al contenido que será impartido para que se despierte su interés por aprender.

Frente a esta realidad, Reveles (2017) afirma que el docente actual debe ser un facilitador en el proceso de aprendizaje. Él no debe concentrarse solamente en enseñar, sino en motivar a su alumno a aprender. Reveles (2017) complementa diciendo que, si bien existe conciencia de eso, aún existen docentes que piensan que su tarea es solamente enseñar, y si el alumno no aprende, es su problema. Estos olvidan que una buena docencia implica escoger las estrategias adecuadas para mejorar el proceso de enseñanza y aprendizaje con un foco centrado en la autonomía del alumno.

Uno de los objetivos de la educación superior es lograr una metodología en que el alumno aprenda a aprender, que pueda desarrollar su capacidad de relacionar conceptos y elaborar una síntesis. A la vez, proporcionar instrumentos para que el estudiante pueda razonar y, de esa forma, logre interrelacionar hechos y obtener conclusiones válidas. Por supuesto que en la educación no solo se trata de aplicar métodos que han sido experimentados y han sido exitosos en otros momentos. Se hace necesaria la adaptación en función de las características de los alumnos. La experiencia docente tiene un rol fundamental para que el método a ser utilizado sea el más apropiado. Acerca de esto, Castillo y Cabrerizo (2005, p. 20) comentan que "se puede afirmar que un profesor utiliza un buen método en tanto en cuanto que lo conoce y que lo sabe ajustar a las características individuales de sus estudiantes". 
Dentro de los métodos activos se encuentra el Aprendizaje Centrado en el Estudiante. Una amplia descripción de esta metodología proviene del Greenwood Dictionary of Education:

La Instrucción Centrada en el Estudiante [ICE] es un enfoque instruccional en el que los estudiantes influyen en el contenido, las actividades, los materiales y el ritmo de aprendizaje. Este modelo de aprendizaje coloca al estudiante (alumno) en el centro del proceso de aprendizaje. El instructor brinda a los estudiantes la oportunidad de aprender de forma independiente y unos de otros y los capacita en las habilidades que necesitan para hacerlo de manera efectiva. El enfoque ICE incluye técnicas como la sustitución de lecciones expositivas por experiencias de aprendizaje activo, la asignación de problemas abiertos y problemas que requieren pensamiento crítico o creativo que no se pueden resolver siguiendo ejemplos de texto, involucrando a los estudiantes en simulaciones y juegos de roles, y utilizando el aprendizaje auto guiado y/o cooperativo (basado en el equipo). La ICE correctamente implementada puede generar una mayor motivación para aprender, una mayor retención de conocimientos, una comprensión más profunda y actitudes más positivas hacia la materia que se enseña. (John Collins y Nancy O'Brien, 2003, citado por Cukierman, 2018, p. 30)

La implementación de un modelo centrado en el estudiante no se produce de un día para el otro. Es necesario capacitar a los docentes para que estén preparados para el cambio que esto exige. Debemos tener en cuenta que algunos docentes, incluso en la carrera de ingeniería, siendo excelentes profesionales en sus actividades, nunca recibieron formación pedagógica y carecen de la capacitación necesaria. Y si la recibieron, probablemente tendría un énfasis en el enfoque tradicional, en el cual el facilitador es quien planifica, desarrolla y evalúa todo el proceso. Por tanto, Cukierman (2018) considera que este enfoque requiere desaprender el rol tradicional para aprender esta forma innovadora en la que el estudiante ocupa el lugar central. Sin embargo, los ingenieros no ofrecerán gran resistencia al cambio, ya que son profesionales que desarrollan, dentro de sus actividades, muchas de las competencias que se promueven en el ACE, por ejemplo:
- aprender de forma autónoma;

- trabajar en equipo;

- aprender junto con sus compañeros.

Esto puede ser muy útil al momento de la implementación del ACE, ya que los docentes ingenieros conocen los beneficios que traen esas competencias en sus vidas laborales, y sería algo muy deseable que los alumnos las puedan desarrollar desde la etapa universitaria. Sin embargo, este cambio no ocurre de forma autónoma. Por eso es de suma importancia que las instituciones de enseñanza estén dispuestas a implementar este tipo de enfoque, que estimulen el cambio con actividades de formación y soporte docente, con el objetivo de que la transformación vaya ocurriendo de forma natural y en un plazo razonable. Sin este apoyo, serán pocos los docentes que se motiven a implementar un modelo basado en el ACE.

Castillo y Cabrerizo (2005) consideran que una metodología fundamentalmente activa es la que debe ser implementada por el docente para proporcionar una mayor participación del estudiante. Si el docente durante el dictado de sus clases suele conducir a sus alumnos a una actitud pasiva, no generará en ellos el interés por lo que están aprendiendo. Los autores enfatizan que:

¡Lo que el estudiante hace, elabora, transforma, experimenta, etc., le es más provechoso que lo que escucha, le dicen o simplemente lee! En el método de enseñanza hay que tener en cuenta, también, la necesidad de partir de hechos concretos a abstractos, de lo conocido a lo desconocido, a utilizar pequeños pasos que desgranen el contenido de la materia,... Son algunas de las estrategias metodológicas con las que el profesor puede facilitar el aprendizaje de los estudiantes. (p. 21)

El ACE sugiere que en determinadas ocasiones el profesor se convierta en una especie de mentor-facilitador educativo, un acompañante que guie el aprendizaje de sus alumnos, creando así un ambiente de confianza donde el estudiante pueda ocupar el protagonismo de su proceso de enseñanza/aprendizaje con una participación activa. Por su parte, el rol del docente será de mentor-facilitador para estimular la autonomía, la criticidad y la creatividad. El profesor solo aportará información y guía cuando sea necesario para que este avance. 
La palabra mentor surge por primera vez en la mitología griega en la obra la Odisea de Homero. Esta palabra hace referencia a un consejero, a una persona con la capacidad de dar consejos (Martínez, 2019). Si trasladamos este concepto a la educación, podemos decir que la mentoría consiste en una relación entre una persona con más experiencia (mentor/docente) y una que carece de la misma (alumno).

Por lo tanto, el ACE considera que estos dos roles (el mentor y el alumno) deben estar bien definidos y que interactúen en un proceso en el cual el docente toma la posición de facilitador, de esta manera el alumno se transforma en el verdadero protagonista de su aprendizaje. La persona con mayor experiencia sirve como guía a la otra para alcanzar el desarrollo de su potencial y la obtención de mejores resultados. No hace falta que este modelo se lleve a cabo en todo momento, se puede implementar en actividades o proyectos específicos, siendo esos momentos definidos por el profesor, basándose en el conocimiento que el mismo tiene de sus estudiantes y del contenido a ser compartido.

Podemos citar como algunas de las características de un mentor (Ureta, 2009):

- Ser una persona con carácter inspirador

- Ser capaz de motivar a su alumno

- Ser humilde

- Tener una escucha activa

- Ser capaz de construir un ambiente de confianza

- Aceptar los errores y verlos como forma de aprendizaje

- Ofrecer un feedback continuo

Cuando decimos que el docente debe tornarse mentor-facilitador de sus alumnos y no ser el centro del proceso de aprendizaje, no significa que este deba dejar de lado la responsabilidad de sus clases, o de explicar conceptos fundamentales a los alumnos en una lección magistral cuando sea necesario. Lo que se está sugiriendo es que el profesor vaya cambiando sus concepciones en cuanto a su rol protagónico y que este lugar sea asumido por sus estudiantes. Las clases deben seguir bajo el cuidado de los docentes y los contenidos que necesiten de su explicación experta serán expuestos por él, pero definitivamente la forma de enseñanza debe ser cambiada, lo cual tornará el proceso de aprendizaje más placentero y efectivo.

Cukierman (2018) destaca que el ACE permite al estudiante tener mayor autonomía durante el tiempo de aprendizaje y consolida el desarrollo de competencias, así como el pensamiento crítico, el trabajo colaborativo, aprendizaje autónomo y autorregulado, entre otras. Cukierman (2018) cita distintos estudios que demuestran esto (J. Lea, Stephenson y Troy, 2003; Brown Wright, 2011; Stanford Center for Opportunity Policy in Education, 2014). Aunque no podemos considerar que este enfoque sea la solución para todos los problemas que tenemos en la educación, pero en ingeniería está ganando mucha importancia en los últimos tiempos. Eso se ve reflejado en el estudio publicado por la Dra. Graham, en el cual destaca:

[...] el surgimiento de una nueva generación de líderes en educación en ingeniería que ofrece currículos integrados centrados en el estudiante a escala. [...] Los entrevistados también sugirieron que, a largo plazo, algunos de los principales programas de ingeniería del mundo ofrecerán cada vez más el aprendizaje centrado en el estudiante a grandes grupos de estudiantes a través de una combinación de aprendizaje en línea personalizado fuera del campus (no presencial) y aprendizaje experiencial "hands-on" en el campus (presencial). (Graham, citado por Cukierman, 2018, p. 31).

\section{Estilos de aprendizaje}

Cuando hablamos de enfoque educativo centrado en el estudiante debemos tener en cuenta la individualidad del alumno en el proceso de aprendizaje. Es importante resaltar que cada individuo posee sus propias perspectivas, experiencias previas, intereses, talentos, capacidades y necesidades. Para esto el docente debe entender un poco más la realidad de sus alumnos. El profesor debe conocer a sus estudiantes, de esta manera podrá asumir su rol como el "docente que explica" o el "mentor que facilita". Por más que veamos a nuestros alumnos parecidos, sea por sus edades o por su nivel académico, raramente encontraremos a dos alumnos iguales. Por esa razón se resalta la importancia que reviste para el docente el hecho de planificar sus clases y personalizar el aprendizaje teniendo en cuenta las características individuales de cada estudiante. 
Los estudiantes aprenden de distintas maneras, algunos son más auditivos, otros más visuales. Están también aquellos que aprenden más fácilmente reflexionando mientras que otros aprenden más cuando actúan. Felder y Silverman (1988) llevaron a cabo una investigación y explican detalladamente este tema. Es muy importante que los docentes tengan presente los estilos de aprendizaje para luego implementar estrategias centradas en ellos de acuerdo a las características comunes que posee cada clasificación.

De una forma resumida, los estilos de aprendizaje están clasificados/divididos en 4 dimensiones y cada dimensión posee dos estilos.

Felder y Silverman (1988) también desarrollaron un test compuesto por 44 preguntas mediante el cual se puede identificar el estilo de aprendizaje de cada persona. Este test se puede encontrar fácilmente en Internet y es una excelente herramienta para que el docente pueda conocer más a sus alumnos y también adaptar sus recursos y metodologías para sus clases. Es imprescindible explicar a los estudiantes sobre esta clasificación proporcionada por los estilos de aprendizaje para que colaboren en la identificación de sus propios estilos y convencerles que al conocerse a sí mismos, pueden lograr un mayor provecho en su proceso de aprendizaje.

Al llevar a cabo la etapa inicial de conocimiento de los estudiantes a través del test de los estilos de aprendizaje, información que resultará de valor tanto para ellos como para los docentes, puede que nos encontremos con algunos obstáculos para implementar estrategias en las cuales el estudiante sea el centro del proceso. Existen algunos autores como, por ejemplo, Campbell y Kryszewska (1992), que afirman que pueden existir tres tipos de problemas al momento de aplicar el Aprendizaje Centrado en el Estudiante.

El primero está relacionado con los alumnos. Existen estudiantes que creen que solamente pueden aprender a través de un profesor, o que están predispuestos a ser cooperativos o, incluso, tienen una baja autoestima. Esta última condición atenta contra la posibilidad de asumir una mayor responsabilidad en el proceso de aprendizaje.

El segundo problema lo representan los docentes que se niegan a asumir un nuevo rol debido a que no están abiertos al cambio, o porque no recibieron ningún tipo de capacitación por parte de las instituciones de enseñanza donde trabajan, o, incluso, porque podrían ser cuestionados en su rol docente.

Por último, están los problemas institucionales, o sea, aquellos que son ajenos a los docentes, como, por ejemplo, los generados por un programa demasiado extenso o estricto que hay que cumplir y el clásico temor por los exámenes finales que son elaborados por otros profesores. O como ya hemos dicho, instituciones que no se ocupan de renovar sus planes de estudio y no persiguen la innovación educativa para sus docentes.

Tabla 7. Estilos de Aprendizaje (Fuente: Felder y Silverman, 1988)

\begin{tabular}{|l|l|l|}
\hline \multicolumn{1}{|c|}{ DIMENSIÓN } & \multicolumn{1}{|c|}{ ESTILOS } & \multicolumn{1}{c|}{ DESCRIPCIÓN } \\
\hline Procesamiento & Activo & Prefiere la experimentación. No aprende mucho en situaciones de pasividad y trabaja bien en grupo \\
\cline { 2 - 3 } & Reflexivo & Necesita pensar sobre la información que se le presenta, trabaja mejor solo y tiende a ser teórico. \\
\hline Percepción & Sensitivo & $\begin{array}{l}\text { Resuelve problemas comprendiendo los métodos. Es bueno memorizando hechos, es cuidadoso } \\
\text { pero lento. }\end{array}$ \\
\cline { 2 - 3 } & Intuitivo & $\begin{array}{l}\text { Prefiere principios y teorías. Le disgusta la repetición. Es bueno para asimilar nuevos conceptos. } \\
\text { Es rápido pero descuidado. }\end{array}$ \\
\hline \multirow{2}{*}{ Entrada } & Visual & Recuerda mejor lo que ve (figuras, diagramas, cuadros, líneas de tiempo, demostraciones, etc.) \\
\cline { 2 - 3 } & Verbal & Recuerda más lo que escucha, aprende a partir de la discusión, y prefiere las explicaciones verbales. \\
\hline Comprensión & Secuencial & Aprende en un orden de progresión lógica regido por el tiempo y el calendario. \\
\cline { 2 - 3 } & Global & $\begin{array}{l}\text { Puede pasar días sin entender un simple problema hasta que de repente logra una rápida comprensión } \\
\text { del todo. }\end{array}$ \\
\hline
\end{tabular}


Implementación del Aprendizaje Centrado en el Estudiante

En este apartado se describirán actividades y recursos del aprendizaje centrado en el estudiante que se implementan en una materia del curso de Ingeniería en Sistemas de Información, en la Facultad Regional de Buenos Aires de la Universidad Tecnológica Nacional (UTN). Actualmente, en la UTN existen diversos docentes que ya están implementando estas y otras estrategias para mejorar el proceso de enseñanza/aprendizaje.

Como explicamos anteriormente con respecto a los problemas que pueden presentarse al implementar el ACE, casi todos los años nos encontramos con alumnos que muestran resistencia a esta metodología y sus estrategias, puede ser que sean alumnos activos o reflexivos que prefieren una enseñanza más tradicional por su mismo estilo de aprendizaje. Sin embargo, a medida que pasan las clases, ellos van comprobando que un ACE los beneficia mucho más que una cursada con modelos donde el docente es el centro de atención. Los alumnos no solo asimilan rápidamente este modelo, sino que también terminan comentando o reclamando cuando pasa cierto tiempo sin aplicar algunas de las estrategias y actividades que utilizamos.

En cuanto a los docentes, en los cursos de capacitación para profesores de la UTN, se puede notar que en el primer encuentro todos tienen temores muy parecidos. Muchas veces se evidencia que se sienten temerosos en el tono de sus preguntas. Con el pasar de las clases, al mostrarles herramientas y recursos que ellos pueden implementar, se nota que su entusiasmo va ganando fuerza. Al finalizar los cursos de capacitación para docentes que ofrece la UTN, los profesores salen con el ánimo renovado, con muchas ganas de implementar lo que fue visto. Aunque debemos saber que la felicidad y el éxito están un paso más allá de nuestra zona de confort, por tanto, es necesario un esfuerzo para lograr el cambio.

\section{Estrategias, actividades y recursos del ACE llevadas a cabo en un curso de Ingeniería en Sistemas de Información}

Discusión entre pares: Existen distintas formas de implementar esta actividad. Una de las más sencillas es presentar a los alumnos una pregunta (o más) de multiple choice referente a un tema ya visto. Puede ser el contenido que acaban de ver o de clases anteriores. Para poder medir las respuestas de forma más rápida y eficiente, se sugiere utilizar aplicaciones de encuestas on-line para teléfonos celulares como, por ejemplo, Socrative (https://www.socrative.com), Poll Everywhere (https://www. polleverywhere.com) o Kahoot (https:// kahoot.com). Se les puede pedir a los alumnos que la bajen previamente. Una vez contestadas las preguntas, los estudiantes deben discutir el problema con sus compañeros por unos minutos y responder nuevamente. (Mazur, 1996)

Aprendizaje Invertido: Pedir a los alumnos que accedan a un determinado material, que puede ser un texto, un video o realizar una breve investigación. Es preferible que el material o tarea solicitada sea corta. Decir al alumno que lea 30 páginas sería arriesgado, así como mirar un video extenso. Al llegar al aula se pueden realizar breves debates o hacer preguntas. Una forma muy efectiva para las preguntas es utilizar herramientas como Socrative (u otras citadas anteriormente), ya que se puede comprobar el resultado de su comprensión durante la clase y compartirlo con los alumnos. (Bergmann y Sams, 2012).

P-D-C (Pensar - Discutir - Compartir): La actividad dura aproximadamente 5 minutos. Les hacemos una pregunta sobre algo que fue visto previamente o algún tema que servirá de introducción a un nuevo contenido. En el primer minuto, cada estudiante de forma individual debe pensar en una respuesta para la pregunta y escribirla en una hoja. En el segundo minuto los estudiantes en pareja discuten sus respuestas pudiendo generar una nueva respuesta en conjunto. Finalmente, cada alumno podrá compartir su respuesta o una síntesis de las conclusiones alcanzadas. Si en el aula hay muchos alumnos se pueden tomar algunas respuestas y discutirlas entre todos. Esta simple actividad genera un clima de alta participación por parte todos y tarda poco más de 5 minutos en ser ejecutada. (Lyman, 1981)

Debates: Plantear dos hipótesis contradictorias sobre determinado tema. Dividir los alumnos en 20 3 grupos (no más de eso) y solicitar que cada grupo elabore argumentos para defender la hipótesis que le fue asignada (más allá de sus conocimientos sobre el tema). Al final los grupos deben debatir entre ellos defendiendo sus argumentos.

\section{Aplicación de actividades y recursos centrados en el estudiante}

Según Bain (2004), los mejores profesores universitarios ayudan a sus estudiantes a pensar sobre la 
información y las ideas. No se dedican solamente a enseñar determinado contenido, sino que buscan la mejor manera para que sus alumnos logren comprender, aplicar, analizar, evaluar evidencias y poder sacar sus propias conclusiones. Para lograrlo, algunos docentes utilizan un método socrático, mientras que otros lo consiguen con la utilización de explicaciones y preguntas, de forma combinada.

En el caso particular de la clase donde hemos implementado las actividades y recursos centrados en el estudiante, previo a la clase, enviamos a los alumnos el material referente al contenido que será visto en el encuentro siguiente. Preferentemente compartimos textos cortos, como, por ejemplo, artículos de revistas, nota del diario o incluso algún video. En el caso de los artículos, tratamos que los textos no superen las 5 páginas, mientras que, en el caso de los videos, que no superen los 15 minutos de duración. Esto estimula al alumno a que lea/vea el contenido. En videos más extensos insertamos preguntas de multiple choice en distintas partes del recurso, las cuales el alumno debe contestar correctamente, ya que forma parte de su evaluación sumativa y no solo formativa. La cantidad de preguntas puede variar según el tiempo que dure el video y también responden a distintos niveles de dominio de las competencias que procuran lograr. De este modo, algunas apuntarán a contenidos conceptuales, otras a procedimientos y otras al desarrollo del pensamiento crítico.

Al iniciar la clase les pedimos a los alumnos que contesten algunas preguntas utilizando el recurso Socrative (https://www.socrative.com) a partir del material enviado previamente. Una vez contestadas las preguntas, nos tomamos 10 minutos para discutir el tema del material que ellos recibieron, de esa forma los estudiantes ya tienen cierto conocimiento del tema y se logra la construcción de lo que vamos a tratar en este día. La herramienta antes mencionada, Socrative, también es utilizada en otros momentos del proceso de aprendizaje, como, por ejemplo, para repasar los contenidos que fueron vistos. Las respuestas que ofrecen los estudiantes posibilitan verificar su nivel de asimilación de los temas y tomar decisiones sobre contenidos que deben ser afianzados.

Otro recurso que utilizamos con mucha frecuencia y con muy buenos resultados en el aula es P-D-C. Este recurso también sirve para introducir un tema nuevo. Antes de comenzar con la actividad correspondiente a ese día, hacemos una pregunta referente a lo que será visto o planteamos un razonamiento que amerite una respuesta. Este ejercicio ofrece una idea del nivel de conocimiento que los estudiantes tienen de determinado tema y ayuda a tomar medidas sobre la profundidad que deben alcanzar en la unidad de aprendizaje, dando más énfasis a tal contenido o tratando más superficialmente aquel otro. También se puede utilizar el P-D-C para repasar el contenido visto. Los alumnos que ya han comprendido pueden explicarles a sus compañeros y se genera así un excelente espacio de aprendizaje entre pares.

Veamos, en las Tablas 2 y 3, un breve esbozo de las guías didácticas en que se implementan actividades centradas en el estudiante, con especial énfasis en aquellas que promueven el razonamiento y la construcción de conceptos:

Tabla 2. Guía didáctica de la clase 2 que incluye actividades centradas en el estudiante

\begin{tabular}{|l|l|}
\hline CLASE 2 & Evaluación con preguntas y respuestas del video "light speed" con Socrative (https://www.socrative.com). \\
\cline { 2 - 3 } & Introducción a las comunicaciones \\
\cline { 2 - 3 } & Ejercicio P-D-C \\
\cline { 2 - 3 } & Un modelo para las comunicaciones \\
\hline & Tareas en los sistemas de comunicación \\
\hline & Protocolos y arquitecturas \\
\hline & Ejercicio P-D-C \\
\hline & Introducción al modelo de referencia OSI \\
\hline & Video sobre modelo de referencia OSI \\
\hline & Introducción al modelo de referencia TCP/IP \\
\hline & Aprendizaje invertido: Análisis del video sobre propagación de onda \\
\hline
\end{tabular}


Tabla 3. Guía didáctica de la clase 3 que incluye actividades centradas en el estudiante

\begin{tabular}{|l|l|}
\hline CLASE 3 & Evaluación del video "propagación de onda" con Socrative (https://www.socrative.com). \\
\cline { 2 - 3 } & Transmisión de datos \\
\cline { 2 - 3 } & Ejercicio P-D-C \\
\hline & Introducción - Señal \\
\cline { 2 - 3 } & Ejercicio P-D-C \\
\cline { 2 - 3 } & Introducción - Onda \\
\hline & Transmisión de señales \\
\cline { 2 - 3 } & Factores que afectan la transmisión \\
\hline & Prestaciones \\
\cline { 2 - 3 } & Debate sobre transmisión de señales \\
\cline { 2 - 2 } & Fibra óptica \\
\hline
\end{tabular}

En la clase 2, después de haber introducido algunos conceptos relativos a comunicaciones y antes de abordar el contenido referente a transmisión, hacemos una pregunta a los alumnos: "¿qué es ancho de banda?". En este momento estamos utilizando P-D-C. Ellos tienen 2 minutos para pensar en una respuesta y escribirla. Una vez finalizado este tiempo, les damos 2 minutos más para discutir con el compañero que tienen al lado y llegar a la mejor respuesta para la pregunta. Al finalizar este tiempo, pedimos que dos o tres alumnos expongan sus respuestas. En este momento tratamos de guiarlos a que logren llegar a la respuesta correcta. Basado en sus respuestas, les pedimos que nos hagan una analogía con algo de la vida diaria que podría ayudar a alguien que no sabe qué es ancho de banda a entender mejor el concepto. Si durante la construcción del concepto nadie citó la analogía de la autopista, entonces la ofrecemos, de modo tal que sirva como otro recurso de aprendizaje para ellos, ya que, como dice Bain (2004, p. 131): "[L]os profesores excepcionales (...) ofrecen explicaciones, analogías y preguntas que ayudarán a los estudiantes a comprender conceptos fundamentales y, consecuentemente, a resolver sus propios problemas".

Esta estrategia no lleva más de 10 minutos en ejecutarla, y es una excelente actividad para que los estudiantes relacionen el contenido visto con la vida real $\mathrm{o}$, incluso, que hagan relaciones que les ayuden a fijar el contenido. También trabajan la discusión entre pares cuando buscan la mejor respuesta para la pregunta propuesta. A veces surge el debate, ya que muchas veces algunos alumnos difieren en sus puntos de vista.
Sobre cuál sería el mejor momento para aplicar estas estrategias, la respuesta es que depende del docente, de los estilos y de las necesidades de aprendizaje de los estudiantes. El profesor es el que debe saber el mejor momento para aplicarlas, ya que él es quien tiene el conocimiento del contenido y del nivel de dominio que deben alcanzar sus estudiantes. Muchas veces surge la actividad de P-D-C o debate, aún sin estar en el plan de la clase, porque los alumnos necesitan cambiar de actividad ante contenidos densos, o puede ser debido a una pregunta o inquietud sobre el tema que surge en los mismos estudiantes, este es el verdadero origen del método socrático.

Un gran consejo que puede servir en la implementación es que no todas las estrategias sirven para todo. Depende mucho del perfil de los alumnos que tenemos. Tampoco sirve querer utilizar todos los recursos, porque de seguro el resultado no será el esperado. Los docentes deben saber seleccionar las estrategias, actividades y recursos mejores y más adecuados para utilizar con sus alumnos.

\section{Resultados de la actividad}

Para medir si la metodología aplicada es o fue exitosa, decidimos implementar evaluaciones previas a la clase con el material enviado antes de la misma. En la primera evaluación realizada en la clase 2, utilizando Socrative, aplicamos un cuestionario con 10 preguntas referentes al video por encargo. Los resultados alcanzados por los alumnos presentes fueron: $67 \%$ obtuvieron un puntaje igual o superior a 8 . Solamente $33 \%$ de los alumnos obtuvieron un puntaje igual o inferior a 7, y 6 fue el puntaje más bajo. (Ver la batería de preguntas en el anexo 1) 


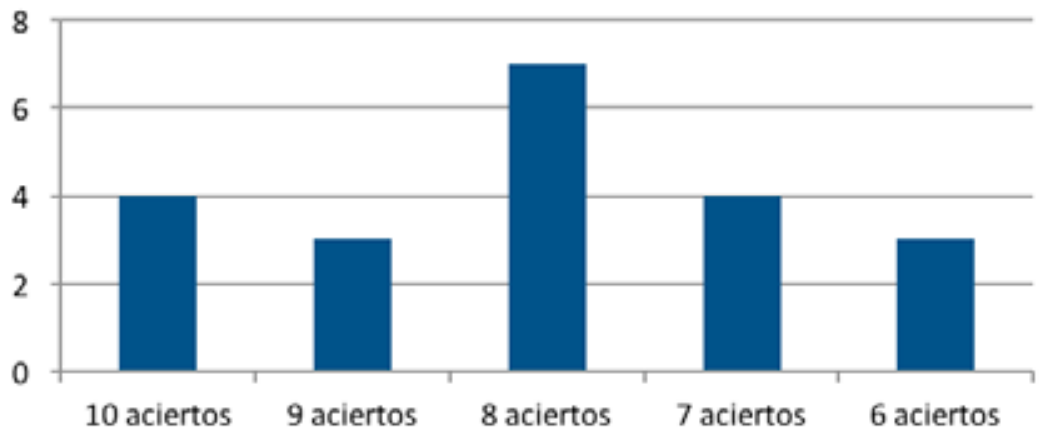

- Cantidad de alumnos

Figura 7. Cantidad de alumnos según la cantidad de aciertos en la actividad propuesta en la clase 2

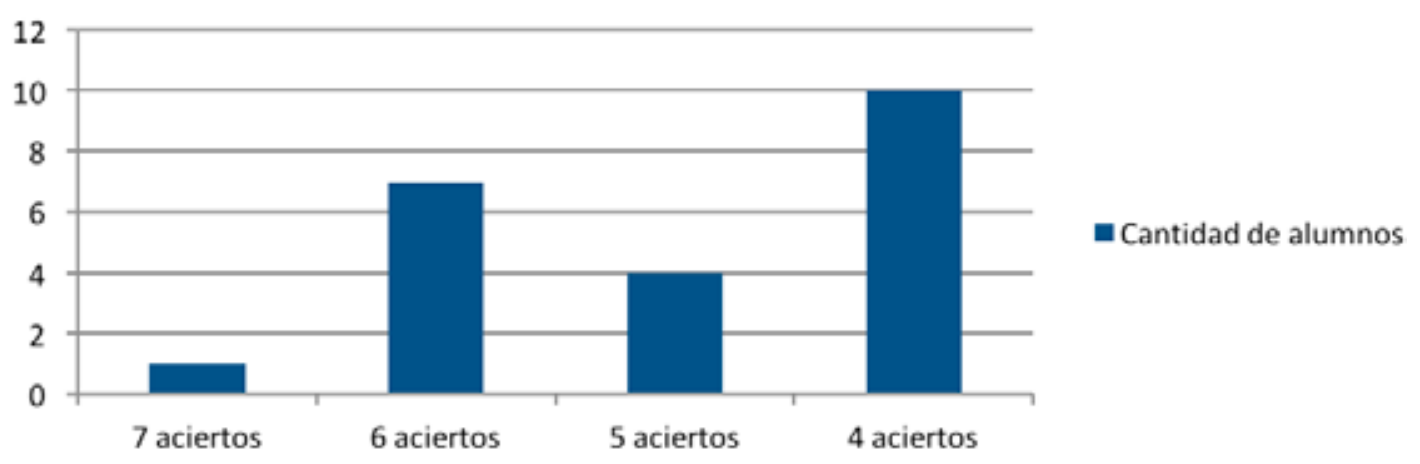

Figura 2. Cantidad de alumnos según la cantidad de aciertos en la actividad propuesta en la clase 3

En la clase 3 también aplicamos la evaluación con Socrative previo a la misma, con preguntas basadas en el vídeo sugerido. Este tenía un grado de complejidad más elevado que el de la clase 2. La actividad estaba compuesta por 7 peguntas. Los resultados fueron los siguientes: $55 \%$ de los alumnos lograron obtener 5 o más aciertos y $45 \%$ de los estudiantes acertaron 4 preguntas. Como mencionamos anteriormente, y teniendo en cuenta que era un material que presentaba mayor complejidad, los resultados siguen siendo alentadores. (Ver la batería de preguntas en el anexo 2)

\section{Conclusiones}

Para un alumno que siempre tuvo la figura del docente como el centro de su proceso de enseñanza/aprendizaje, este tipo de metodología podría causarle cierto asombro. Por supuesto que esta resistencia al cambio es solo al principio, por eso debemos ser lo más transparentes posible con ellos. Siempre desde el primer día de clase debemos compartir con el grupo cómo será la dinámica utilizada durante la cursada, las formas de evaluación, etc. También es aconsejable que entreguemos el programa de la materia, ya que consideramos esto una especie de contrato entre los estudiantes y el docente.

Cuando vamos a utilizar una nueva estrategia en alguna clase, debemos aclararles lo que estamos haciendo y el objetivo que lograremos al mejorar su proceso de aprendizaje. Pero también, debemos ser muy honestos y decirles que podemos equivocarnos. Con esto los alumnos empiezan a entender que esta nueva dinámica tiene como objetivo generar un ambiente de estudio más propicio para el aprendizaje, donde puedan desarrollar mejor las competencias y con eso aprender de una forma más activa. Las preguntas que hacemos a los alumnos al concluir la asignatura puede dar una idea de cómo valoran la clase cuando se aplican estas estrategias o cuando se utilizan nuevas tecnologías.

No debemos tener miedo al cambio ni a probar innovaciones en nuestra tarea. El error es parte del aprendizaje. En mi caso, cuando voy a utilizar una nueva herramienta o técnica, siempre les aclaro a los alumnos que es algo nuevo y que creo que traerá buenos resultados y por eso la utilizaré con ellos. Esto permite una relación más transparente entre profesor y estudiante. Si algo sale mal, si la 
estrategia/técnica no da los resultados esperados, no hay que desanimarse. Seguramente ellos entenderán y no se sentirán frustrados.

Capacitarse constantemente es una de las claves para este éxito. El docente debe mantenerse con la motivación de crecer para desempeñar su rol de manera eficaz. Es decir, estar atento a nuevos materiales, estudios y herramientas que surgen. Sin embargo, tal y como hemos enfatizado, hay que saber escoger adecuadamente lo que se utilizará con los alumnos y conocer su estilo de aprendizaje, ya que no todo sirve para todos. Querer utilizar todas las estrategias y recursos que existen podría resultar complejo y saturar el proceso de enseñanza-aprendizaje en vez de lograr un desarrollo óptimo del mismo. Nosotros como docentes debemos conocer el perfil de nuestros estudiantes para poder, a partir de ese conocimiento, elegir las mejores estrategias, recursos y actividades y así probablemente nuestros estudiantes tendrán un aprendizaje más eficaz y profundo y un mejor desempeño en su vida profesional.

Hay una parte del proceso de aprendizaje que exige la necesidad de repetición y métodos habituales, pero está la otra parte que promueve la innovación y la sorpresa. Un buen maestro logra ofrecer un equilibrio entre lo sistemático y lo innovador. (Bain, 2004).

\section{Referencias bibliográficas}

Bain, K. (2004). Lo que hacen los mejores profesores universitarios. Valencia: Publicacions de la Universitat de Valencia.

Bergmann, J. y Sams, A. (2012). Flip your Classroom: Reach Every Student in Every Class Every Day. ISTE - ASCD.

Bergmann, J. (2018). Por qué el aprendizaje centrado en los estudiantes es sólo la mitad. Revista oficial del II Congreso Europeo Flipped Classroom.

Campbell, C. y Kryszewska, H. (1992). Learner-based Teaching. Reino Unido: Oxford.

Castillo, S. y Cabrerizo, J. C. (2005). Formación del Profesorado en Educación Superior: Desarrollo Curricular y Evaluación (Vol. II). España: McGraw-Hill Interamericana.
Cukierman, U. (2018). Aprendizaje centrado en el estudiante: un enfoque imprescindible para la educación en ingeniería. Recuperado el 04 de Junio de 2019 de Academia.edu: https:// www.academia.edu/37040716/Aprendizaje_ centrado_en_el_estudiante_un_enfoque_ imprescindible_para_la_educaci\%C3\%B3n_en_ ingenier\%C3\%ADa

Escribano, A. y del Valle, A. (2008). El Aprendizaje Basado en Problemas. Una propuesta metodológica en Educación Superior. Madrid: Narcea.

Felder, R. y Silverman, L. (1988). Learning and Teaching Styles In Engineering Education. Journal of Engineering Education, 674-681.

Hernández, J., Salgado, A. y Sandoval, I. (13 de Noviembre de 2007). Planeación para el Aprendizaje Centrado en el Estudiante con Enfoque de Proyectos, un caso de circuitos. Acesso el 16 de junio de 2019, disponíble en Congreso Internacional de Innovación Educativa: https://www.repo-ciie.cgfie.ipn.mx/memorias/II/ mesas1311071.html

López, F. (2007). Metodología Participativa en la Enseñanza Universitaria. Madrid: Narcea.

Lyman, F. (1981). The Responsive Classroom Discussion: The Inclusion of All Students. Maryland: University of Maryland, College Park.

Manzanares, A. y Palomares, M. (2008). Tutoría y Mediación en el Aprendizaje Basado en Problemas. En A. Escribano, y A. del Valle. El aprendizaje Basado en Problemas. Una propuesta metodológica en Educación Superior. Madrid: Narcea.

Martínez, F. (20 de abril de 2019). Obtenido de https:// www.efeemprende.com/blog/eie-palabra-mentor/

Mazur, E. (1996). Peer Instruction: A User's Manual. England: Pearson.

Reveles, J. d. (24 de Diciembre de 2017). Otras Voces en Educación. Recuperado el 24 de Mayo de 2019, de http://otrasvoceseneducacion.org/archivos/261905

Ureta, C. (2009). Competencias del Profesor-Mentor para el Acompañamiento al Profesorado Principiante. Profesorado: Revista de Currículum y Formación del Profesorado, 209-229.

Weimer, M. (2002). Learner-centered teaching: Five key changes to practice. San Francisco, CA: Jossey-Bass. 


\section{Anexo 1}

Preguntas utilizadas en las actividades de las clases 2

Vale aclarar que las preguntas están clasificadas en niveles cognitivos: conocimiento, procedimiento y razonamiento crítico, y no con el orden en que figuran en la herramienta Socrative.

Clase 2

\section{Conocimiento:}

Responde, a continuación, si este gráfico tiene algún error y, si lo hubiera, cuál es el error

¿Qué significa un protocolo y para qué sirve?

¿La amplitud de una señal influye en su frecuencia?

¿La longitud de onda es inversamente proporcional a la frecuencia?

¿Qué es una antena y para qué sirve?

Cuando se transmiten datos digitales por medio de señales digitales, ¿cada elemento de señal transmite un solo bit?

¿En qué consiste la modulación?

¿Qué tipo de multiplexación utiliza el sistema ADSL?

\section{Procedimiento:}

En nuestra vida diaria, ¿cuáles serían los factores que afectan una transmisión?

\section{Razonamiento crítico:}

¿Estás de acuerdo con lo que menciona el video sobre datos digitales? Justifica la respuesta

\section{Anexo 2}

Preguntas utilizadas en las actividades de las clases 3

Vale aclarar que las preguntas están clasificadas en niveles cognitivos: conocimiento, procedimiento y razonamiento crítico y no con el orden en que figuran en la herramienta Socrative.

Clase 3

\section{Conocimiento:}

¿Cuántas capas tiene el modelo de referencia OSI?

¿Qué características tiene una señal periódica?

El espectro de una señal es:

¿Para qué se usa la propagación ionosférica de ondas?

La propagación por trayectoria visual (LOS) de ondas se usa para:

\section{Procedimiento:}

¿Existe una relación entre el espectro de una señal y la propagación superficial de ondas? Justifica la respuesta

\section{Razonamiento crítico:}

¿Estás de acuerdo sobre lo comentado en el video sobre la señal? Justifica tu opinión 